\title{
Plastic flow of persistent currents in two dimensional strongly interacting systems
}

\author{
Richard Berkovits and Yshai Avishai ${ }^{\dagger}$ \\ The Minerva Center for the Physics of Mesoscopics, Fractals and Neural Networks, \\ Department of Physics, Bar-Ilan University, Ramat-Gan 52900, Israel \\ †also at Department of Physics, Ben-Gurion University, Beer-Sheva, Israel
}

(August 8, 2018)

\begin{abstract}
The local persistent current in two dimensional strongly interacting systems is investigated. As the interaction strength is enhanced the current in the sample undergoes a transition from diffusive to ordered flow. The strong interacting flow has the characteristics of a plastic flow through dislocations in the pinned charge density wave which develops in the system at low densities.
\end{abstract}

PACS numbers: 71.55.Jv,71.27.+a,73.20.Dx

Typeset using REVTEX 
The behavior of interacting electrons in random potentials has drawn much interest because of its relevance to many different phenomena such as persistent currents [1], the two-dimensional MIT (metal-insulator transition) [2], and the charging spectra of quantum dots 3 [5]. It is generally believed that the large amplitudes of persistent currents measured for mesoscopic rings in the diffusive regime [6:7] are the result of the suppression of the influence of disorder by interactions, although no quantitative calculation for a realistic model of a 3D metallic system has been forthcoming.

Much recent work has concentrated on the influence of interactions on 2DEG (2D Electron Gas). It is speculated that the experimentally observed 2D MIT [2] is due to e-e (electron-electron) interactions [8], while indications of the influence of e-e interactions in the addition spectrum of disordered quantum dots are mounting [3, [, F. For typical 2DEG devices the density is rather low $\left(n<3 \times 10^{11} \mathrm{~cm}^{-1}\right)$ which corresponds to a ratio between the the Coulomb and Fermi energy $r_{s}=e^{2} / v_{f}>1$ (where $v_{f}$ is the Fermi velocity) [9]. In this region the simple RPA (random phase approximation) no longer holds, and correlations play an important role [9].

Persistent currents for a clean 2DEG ring has been recently measured and shown to be of the expected magnitude [10]. No measurements have yet been performed in the diffusive regime, but numerical calculations indicate that the persistent current will be significantly enhanced (compared to the non-interacting value) by the e-e interactions even for spinless electrons [11].

In this paper we would like to clarify the mechanism of this enhancement in 2DEG rings. A useful clue may be obtained from the study of the distribution of the current in different realizations of disorder [12]. While in the non-interacting case the distribution is broad with almost the same probability for dia or paramagnetic currents leading to small paramagnetic average currents, in the interacting case the distribution is predominately paramagnetic leading to large paramagnetic average currents. In this paper we shall show that the local persistent current undergoes a transition from a diffusive behavior for the non-interacting regime to a strongly correlated plastic flow through a small number of channels for stronger 
e-e interactions. This leads to a change in the local distribution of the current and to the enhancement of the total average current.

Thus, there are two distinct mechanisms for the enhancement of the persistent current in 2DEG systems. One which is relevant to the high density weak interaction regime which is described analytically by the diagrammatic perturbation treatment [13, 14], or by the Hartree-Fock picture [15], and treated numerically by various Hartree-Fock approximations [16 19]. The other is the low density strongly interacting regime which is characterized by the appearance of correlations in the electronic density and plastic flow of the persistent current.

The local persistent current of an interacting 2D cylinder of circumference $L_{x}$ and height $L_{y}$ threaded by a flux $\Phi$ was calculated. This system is known to show a large enhancement of the persistent current in the diffusive regime [11]. The Hamiltonian is given by:

$$
H=\sum_{k, j} \epsilon_{k, j} a_{k, j}^{\dagger} a_{k, j}-i \sum_{k, j} J_{k, j}^{x}+J_{k, j}^{y}+U \sum_{k, j>l, p} \frac{a_{k, j}^{\dagger} a_{k, j} a_{l, p}^{\dagger} a_{l, p}}{\left|\vec{r}_{k, j}-\vec{r}_{l, p}\right|}
$$

where

$$
\begin{aligned}
& J_{k, j}^{x}=-i V \exp \left(i \Phi s / L_{x}\right) a_{k, j+1}^{\dagger} a_{k, j}-\text { h.c, } \\
& J_{k, j}^{y}=-i V a_{k+1, j}^{\dagger} a_{k, j}-\text { h.c, }
\end{aligned}
$$

and $a_{k, j}^{\dagger}$ is the fermionic creation operator, $\epsilon_{k, j}$ is the energy of a site $(k, j)$, which is chosen randomly between $-W / 2$ and $W / 2$ with uniform probability, $V$ is a constant hopping matrix element and $s$ is the lattice constant. The distance $\left|\vec{r}_{k, j}-\vec{r}_{l, p}\right|=\left(\min \left\{(k-l)^{2},\left(L_{x} / s-(k-\right.\right.\right.$ $\left.\left.l))^{2}\right\}+\min \left\{(j-p)^{2},\left(L_{y} / s-(j-p)\right)^{2}\right\}\right)^{1 / 2}$. The interaction term represents a Coulomb interaction between electrons confined to a $2 \mathrm{D}$ cylinder embedded in a 3D space with $U=$ $e^{2} / s$,

We consider a $4 \times 4$ lattice with $m=16$ sites and $n=8$ electrons. The many-particle Hamiltonian then may be represented by a $12870 \times 12870$ matrix, which is exactly diagonalized. The many-particle ground state $|\Psi(\Phi)\rangle$ for $\Phi=\pi / 2$ is calculated for 500 different realizations of disorder in the diffusive regime $(W=8 \mathrm{~V}$ [11]) for several values of interaction $U$. The local persistent current 


$$
I_{k, j}^{a}(\Phi)=\left\langle\Psi(\Phi)\left|J_{k, j}^{a}\right| \Psi(\Phi)\right\rangle
$$

(where $a=x, y$ ) is calculated for each realization.

Local currents in typical realizations are plotted in Fig. 1. It can be seen that the current for the non-interacting case $U=0$ is diffusive, the current flows in all directions, there are current loops and there is no obvious long range correlations. On the other hand for $U=10 \mathrm{~V}$, which corresponds to $r_{s} \sim \sqrt{\pi / 2}(U / 4 V) \sim 3$ a value which could be easily obtained in contemporary $2 \mathrm{DEG}$ devices, the behavior is totally different. The persistent current flows through a few channels in the sample, there are no currents flowing in the opposite directions, no closed loops, and obvious correlations. This situation reminds us of plastic flow in lattices, for example the flow of magnetic vertexes in driven Abrikosov lattice pinned by random pinning centers [20].

Indeed for interaction strength corresponding to $r_{s}>1$ the system described by the Hamiltonian in Eq. (1) is known to exhibit short range density correlations which develop into a CDW (charge density wave) for stronger interactions [9]. The charge density and the persistent current for some typical realization are shown in Fig. 2. Most of the current flows in channels which correspond to dislocations in the pinned charge density wave clearly seen in the density plot of the different realizations. This is a feature which is characteristic of plastic flows.

A more quantitative measure for the change that the persistent current in the sample undergoes due to the e-e interactions is given by the correlation between the currents at different locations. This correlation may be formulated in the following way:

$$
C(r, \Phi)=\frac{\sum_{k, j}\left\langle I_{k, j}^{x}(\Phi) I_{k, j+r}^{x}(\Phi)\right\rangle-\left\langle I^{x}\right\rangle^{2}}{\left\langle\left(I^{x}(\Phi)\right)^{2}\right\rangle-\left\langle I^{x}(\Phi)\right\rangle^{2}}
$$

where

$$
\left\langle\left(I^{a}(\Phi)\right)^{N}\right\rangle=\left\langle\left(I_{k, j}^{a}(\Phi)\right)^{N}\right\rangle
$$

and $\langle\ldots\rangle$ denotes average over different realizations of disorder. The average current $\left\langle I^{a}(\Phi)\right\rangle$ as well as the typical current $\sqrt{\left\langle\left(I^{a}(\Phi)\right)^{2}\right\rangle}$ on a bond are plotted in the inset of Fig. 3, while 
the current per bond distribution $P\left(I^{x}\right)$ for different values of interaction strength are plotted in Fig. 3. As in the case of the total average current shown in Ref. [11 the average current in the $\hat{x}$ direction increases up to $U=10 \mathrm{~V}$ and then decreases, while the typical current is somewhat enhanced up to $U=10 \mathrm{~V}$ and then decreases. Of course the average current in the $\hat{y}$ direction is zero and the typical current is somewhat enhanced in the weak interaction regime $\left(r_{s}<1\right.$ corresponding to $U<\sqrt{32 / \pi} \sim 3$, but suppressed for higher values of interaction.

The average current is strongly enhanced in the regime of $r_{s}<3$. It is clear from the typical bond current dependence on $U$ and from the distribution that the enhancement is not due to an enhancement of the typical current in a certain realization but due to the fact that in the interacting case the local current in the $\hat{x}$ direction for almost all realizations is paramagnetic while in the non-interacting case there is an almost equal probability of the current being para or diamagnetic.

The correlation between the persistent currents at neighboring bonds as function of the interaction strength is seen in the plot of $C(r=1, \Phi=\pi / 2)$ presented in Fig. 4 . While in the weak interaction RPA regime there is no enhancement of the local current correlations due to the increase in the interaction strength, there is a strong enhancement of these correlations in the strong interaction low density regime $r_{s}>1$. Thus, the impression one gets from the current plots shown in Figs. 1 and 2 of a transition in the current characteristics of the sample, is confirmed.

The reason for the enhancement of the average total current becomes now quite clear. While in the weak interaction RPA regime there is some enhancement in the average current due to interactions, this enhancement is not related to correlations in the local current. On the other hand, in the strongly interacting low density regime the enhancement is connected to an ordering of the local persistent current. The local persistent current in the strongly interacting case must flow along dislocation lines. There is only a small probability of forming closed loops and changing directions resulting in the fact that the current in most realizations flows in the same direction and the distribution of the current is almost exclusively 
paramagnetic.

In conclusion, strong interactions (or low densities) impose a significant modification in the nature of the local persistent current in a $2 \mathrm{D}$ sample. The current is transformed from a diffusive current to a plastic flow along dislocations of the emerging pinned CDW of the system. This transformation causes a strong enhancement in the total persistent current of the system since there is no suppression of the average current due to the fact that in different realizations current might flow in arbitrary directions.

We are grateful to the Israeli Academy of Sciences and Humanities research center "Strongly Interacting Electrons in Restricted Geometries" for financial support. 


\section{REFERENCES}

[1] For a recent review see: T. Guhr, A. Müller-Groeling and H.A. Weidenmüller, Phys. Reports (in press, cond-mat/9707301), and reference therein.

[2] S. V. Kravchenko et. al. Phys. Rev. Lett. 77, 4938 (1996); D. Popovic, A. B. Folwer and S. Washburn, Phys. Rev. Lett. 79, 1543 (1997).

[3] U. Sivan, R. Berkovits, Y. Aloni, O. Prus, A. Auerbach and G. Ben-Yoseph, Phys. Rev. Lett. 77, 1123 (1996).

[4] F. Simmel, T. Heinzel and D. A. Wharam, Europhys. Lett. 38, 123 (1997).

[5] S. R. Patel, S. M. Cronenwett, D. R. Stewart, A. G. Huibers, C. M. Marcus, C. I. Duruoz, J. S. Harris, K. Campman and G. Gossard, (preprint cond-mat/9708090).

[6] L. P. Levy, G. Dolan, J. Dunsmuir, and H. Bouchiat, Phys. Rev. Lett. 64, 2074 (1990).

[7] V. Chandrasekhar, R. A. Webb, M. J. Brady, M. B. Ketchen, W. J. Galager and A. Kleinsasser, Phys. Rev. Lett. 67, 3578 (1991).

[8] V. Dobrosavljevic, E. Abrahams, E. Miranda, and S. Chakravarty, Phys. Rev. Lett. 79, 455 (1997); C. Castellani, C. DiCastro and P. A. Lee cond-mat/9801006).

[9] R. Berkovits and B. L. Altshuler, Phys. Rev. B 55, 5297 (1997).

[10] D. Mially, C. Chapelier and A. Benoit, Phys. Rev. Lett. 702020 (1993).

[11] R. Berkovits and Y. Avishai, Europhys. Lett. 29, 475 (1995); R. Berkovits and Y. Avishai, Phys. Rev. Lett. 76, 291 (1996).

[12] R. Berkovits and Y. Avishai, Solid State Commun. 93, 301 (1995).

[13] V. Ambegaokar and U. Eckern, Phys. Rev. Lett. 65, 381 (1990).

[14] A. Schmid, Phys. Rev. Lett. 66, 80 (1991).

[15] G. Montambaux, J. Phys. I (France), 6, 1 (1996). 
[16] M. Ramin, B. Reulet, H. Bouchiat Phys. Rev. B 51, 5582 (1995).

[17] H. Kato and Y. Yoshika, Physica B 212, 251 (1995).

[18] G. Bouzerar and D. Poilblanc, Phys. Rev. B 52, 10772 (1995).

[19] A. Völker and P. Kopietz, Z. fur Physik B, 102, 545 (1997).

[20] F. Nori, Science 271, 1373 (1996). 


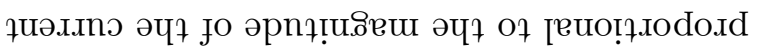

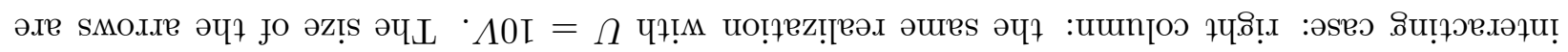

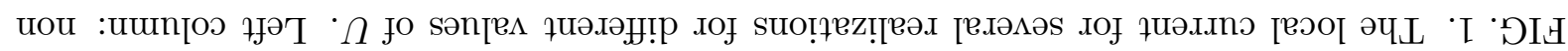
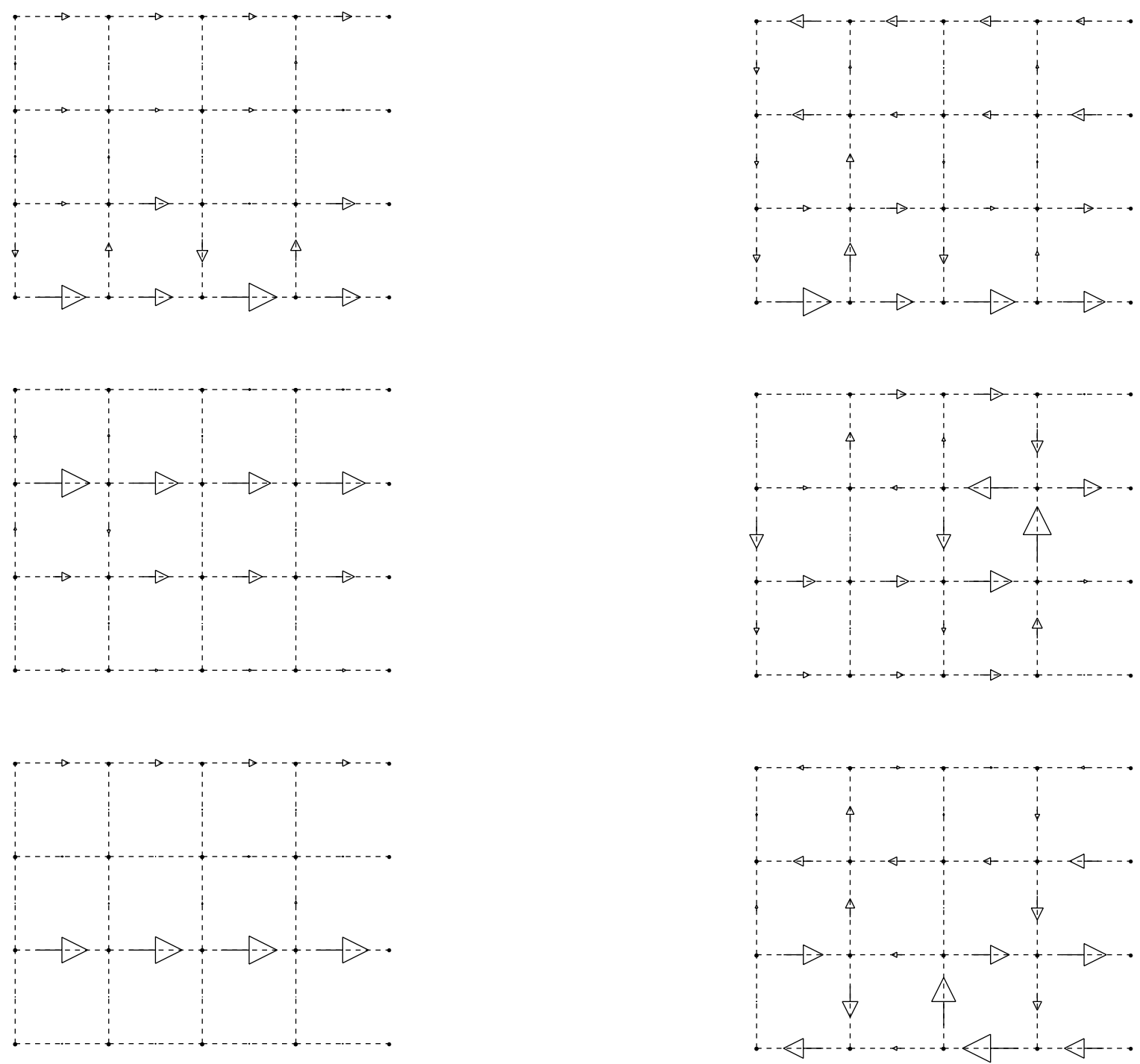

$\Lambda 0 \mathrm{I}=\mathrm{\Omega}$

$0=\Omega$ 


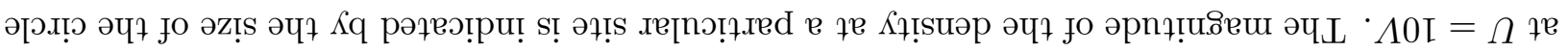

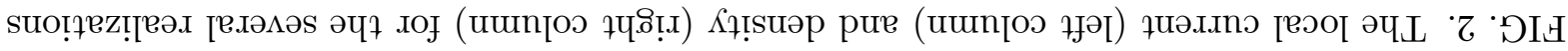
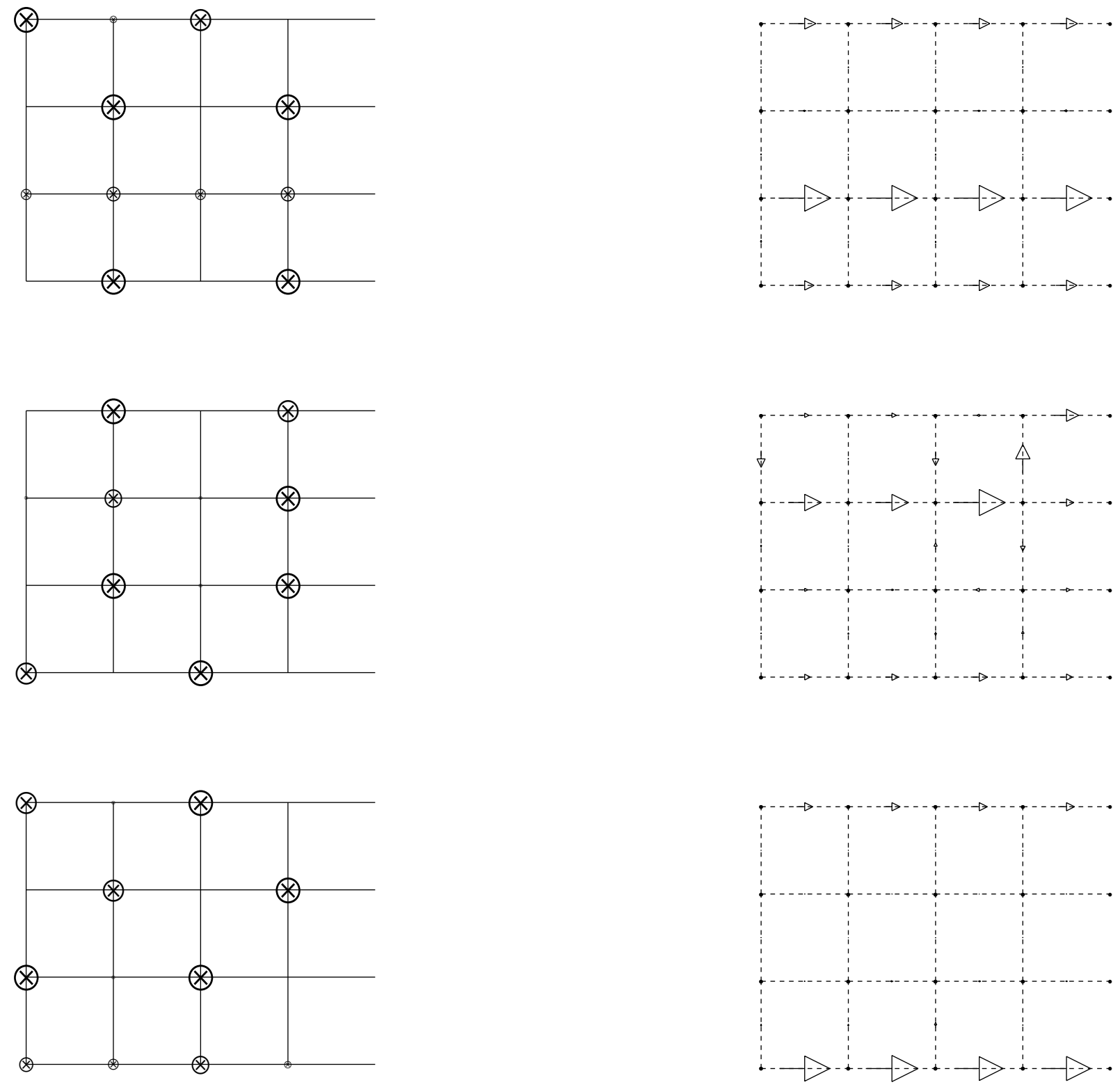


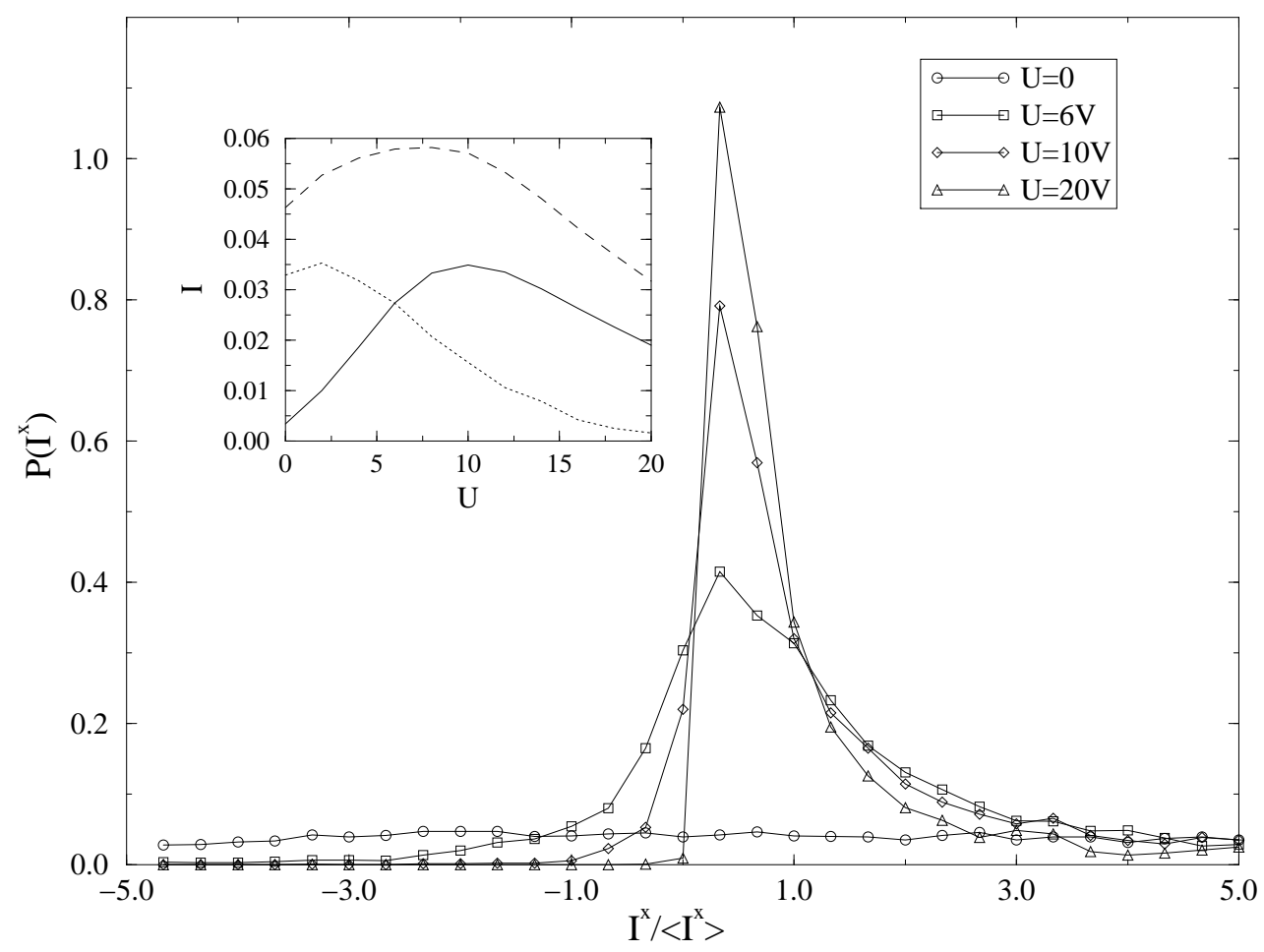

FIG. 3. The distribution of the bond current in the $\hat{x}$ direction for different values of $U$. Inset: average (full line) and typical (dashed line) bond currents in the $\hat{x}$ direction and typical (dotted line) bond currents in the $\hat{y}$ direction in units of the hopping matrix $V$. 


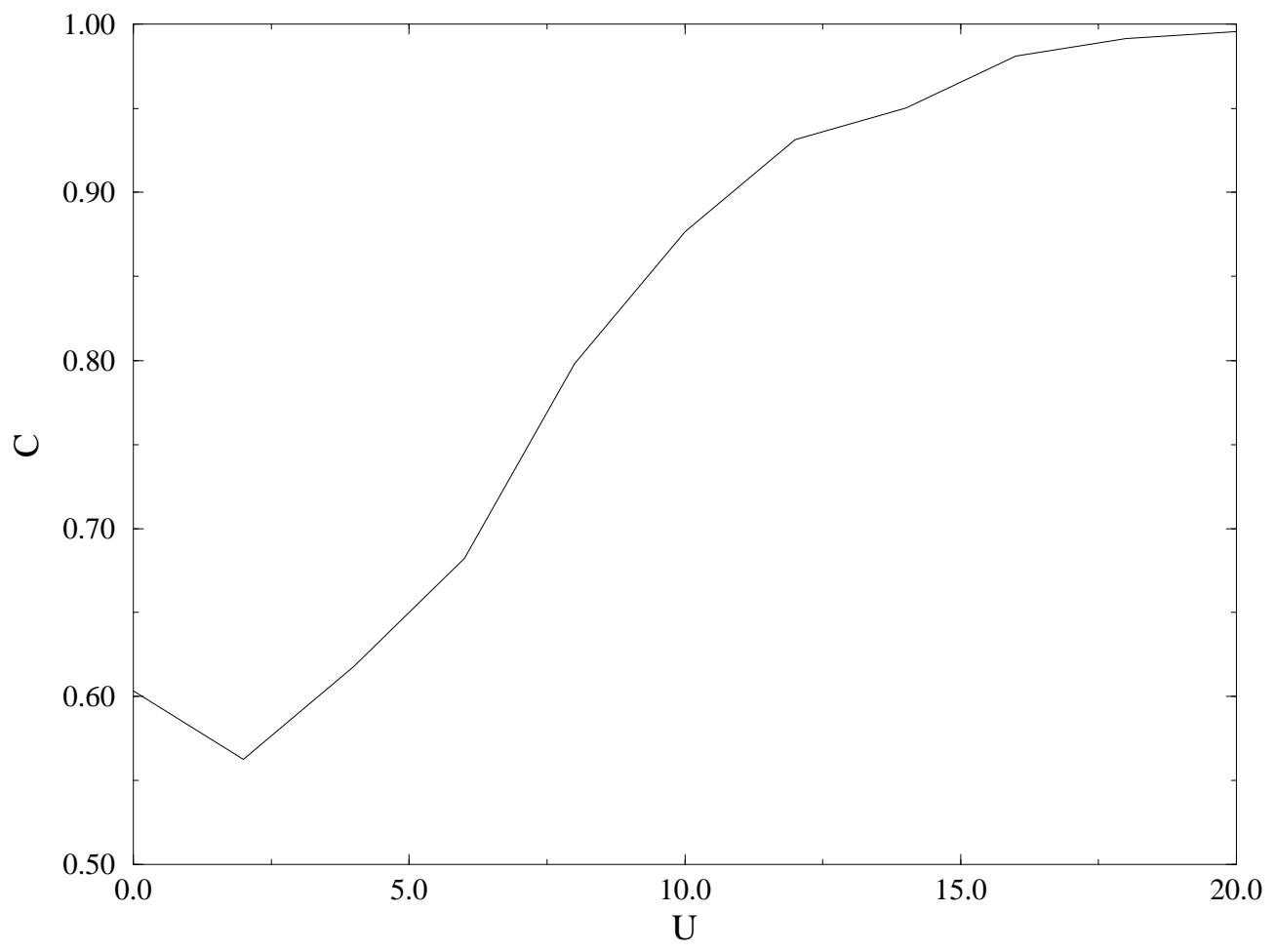

FIG. 4. The bond current correlation in the $\hat{x}$ direction as function of the interaction strength $U$. 